\title{
Fakeons And Microcausality: \\ Light Cones, Gravitational Waves \\ And The Hubble Constant
}

\author{
Damiano Anselmi ${ }^{1,2,3}$ and Antonio Marino ${ }^{1,4}$ \\ ${ }^{1}$ Dipartimento di Fisica "Enrico Fermi", Università di Pisa, \\ Largo B. Pontecorvo 3, 56127 Pisa, Italy \\ and ${ }^{2}$ INFN, Sezione di Pisa, \\ Largo B. Pontecorvo 3, 56127 Pisa, Italy \\ ${ }^{3}$ damiano.anselmi@unipi.it, ${ }^{4}$ antonio.marino7695@gmail.com
}

\begin{abstract}
The concept of fake particle, or "fakeon", allows us to make sense of quantum gravity as an ultraviolet complete theory, by renouncing causality at very small distances. We investigate whether the violation of microcausality can be amplified or detected in the most common settings. We show that it is actually short range for all practical purposes. Due to our experimental limitations, the violation does not propagate along the light cones or by means of gravitational waves. In some cases, the universe even conspires to make the effect disappear. For example, the positivity of the Hubble constant appears to be responsible for the direction of time in the early universe.
\end{abstract}




\section{Introduction}

The idea of fakeon has been put forward in 2017 in refs. [1, 2] to ovecome the problem of ghosts in higher-derivative theories and ensure unitarity. With broader applications, fakeons can be used even in non-higher-derivative theories, for example when a field has a positive squared mass, irrespectively of the sign of the pole of its propagator.

The fakeon is a degree of freedom that can only be virtual. It does not belong to the physical spectrum. It provides a better understanding of the Lee-Wick models [3, 4], overcoming ambiguities [5] and problems with Lorentz invariance [6], and actually leading to the completion of their formulation [7]. Moreover, fakeons allow us to simplify the proofs of perturbative unitarity in gauge and gravity theories [8]. But their most important application is that they lead to a consistent, and basically unique, theory of quantum gravity $[1,9,10]$.

One physical consequence due to the fakeons is the violation of causality at energies larger than their masses. The existence of a relation between higher-derivatives and violations of microcausality has been known for a long time. For example, in classical electrodynamics, the runaway solutions predicted by the Abraham-Lorentz force can be eliminated by renouncing microcausality [11]. An analogous "reduction" can be implemented in quadratic gravity [12]. The Lee-Wick models lead to the violation of microcausality naturally, as realized quite soon $[4,13]$. Without higher-derivatives, a version of electrodynamics that has issues with causality is the Feynman-Wheeler theory [14], which (potentially) involves the classical analogue of a massless fakeon. However, since a massless fakeon implies the violations of both microcausality and macrocausality, Feynman and Wheeler developed a nontrivial "absorber-emitter theory" to annihilate the effects of the potential fakeon and recover causality altogether.

In quantum field theory, a satisfactory definition of causality is lacking [15]. Bogoliubov's condition [16] is off-shell, like the Lehmann-Symanzik-Zimmermann requirement that fields commute at spacelike separated points [17]. At the practical level, the difficulty is to accurately localize spacetime points working with relativistic wave packets that describe on-shell particles. In most cases, we may have to downgrade the violation of causality to an unusual form of the equations [18]. Nonetheless, if the discrepancies with respect to the predictions that follow from the ordinary equations can be confirmed or refuted experimentally, we have a way to make progress, no matter what those discrepancies are supposed to mean. 
The fake degrees of freedom must be projected away, both at the quantum level and classically. An important feature of the theories that contain fakeons is that the starting classical action is not the true classical action [19], but an "interim" local action that provides the basic Feynman rules. The true classical action is nonlocal and can be obtained after the quantization, by means of a process of classicization of the interim action. Forms of violation of microcausality survive the classical limit, due to the presence of certain "fakeon averages" in the projected field equations. This fact suggests that it might be possible to detect the violation experimentally at some point, as a discrepancy with respect to the ordinary equations, or a fuzziness of the initial conditions, the time evolution, etc.

In this paper we study the effects of the fakeons on the classical limit of quantum gravity. Can the violations of microcausality be amplified into violations of causality? Does Lorentz symmetry spread the effects along the light cones? Can the gravitational waves propagate the effects to long distances?

Since the theory is Lorentz invariant, the violation occurs for sufficiently small invariant intervals. Then, it should be possible to propagate it to arbitrary distances, close enough to the light cones, if we wait for a sufficient amount of time. This is true in principle, but has no practical consequences, for reasons related to the poor accuracies of our measurements. In all realistic situations the violation of microcausality remains confined within a radius of order $1 / m$, where $m$ is the fakeon mass. To spread it out, we need sources that oscillate with a frequency $\omega$ of order $m$, which are not realistic, even if we assume that the fakeon masses $m$ are relatively small (say, several orders of magnitude below the Planck mass).

We also find that the impact of fakeons on the gravitational radiation is negligible, which excludes the possibility of propagating the violation of microcausality to longer distances by means of gravitational waves. Again, it would be necessary to generate radiation with very large frequencies.

We conclude that the amplification of the violation of microcausality does not appear to be around the corner. Actually, nature is for some reason keeping it confined down to small distances. For example, we show that the positivity of the Hubble constant conspires to suppress the violation and give time a direction in the early universe.

The approach to quantum gravity based on the idea of fake particle comes from perturbative quantum field theory, so the fakeon prescription is understood at present in momentum space and working perturbatively around flat space. The problem of fully understanding the fakeon prescription in coordinate space (beyond the tree level) or around generic backgrounds is still open at this stage. In some cases, more general backgrounds 
can be reached by means of resummations of the results obtained around flat space. An example of this type is given in section 4 .

The paper is organized as follows. In section 2 we study the fakeon average and discuss its effects close to the light cones. In section 3 we extend the analysis to the gravitational waves. In section 4 we study the effects of the Hubble constant on the fakeon average. Section 5 contains the conclusions and appendix A is devoted to the technical aspects of a calculation.

\section{Light cones and microcausality}

In this section we study the classical limit of the fakeon Green function. In particular, we show that the violation of microcausality does not propagate along the light cones if the source is slowly varying for time intervals of order $1 / m$, where $m$ is the mass of the fakeon.

Consider the Klein-Gordon equation

$$
\left(1+\frac{\square}{m^{2}}\right) \phi(x)=J(x)
$$

where $J$ is a real source and $m$ a mass. If $\phi$ is a fakeon, its solution is [19]

$$
\phi(x)=\langle J\rangle_{\mathrm{f}}(x) \equiv \int G_{\mathrm{f}}(x-y) J(y) \mathrm{d}^{4} y
$$

where $\langle J\rangle_{\mathrm{f}}$ denotes the "fakeon average"

$$
\langle J\rangle_{\mathrm{f}}=\left.\frac{m^{2}}{\square+m^{2}}\right|_{\mathrm{f}} J \equiv \frac{m^{2}}{2}\left(\left.\frac{1}{\square+m^{2}}\right|_{\text {ret }}+\left.\frac{1}{\square+m^{2}}\right|_{\text {adv }}\right) J .
$$

The violation of causality is due to the contributions of the advanced potentials.

In Fourier transforms, we get

$$
G_{\mathrm{f}}(x)=\mathcal{P} \int \frac{\mathrm{d}^{4} p}{(2 \pi)^{4}} \frac{-m^{2} \mathrm{e}^{-i p \cdot x}}{p^{2}-m^{2}}=\frac{1}{2}\left[G_{+i \epsilon}(x)+G_{-i \epsilon}(x)\right],
$$

where $\mathcal{P}$ denotes the principal value and

$$
G_{ \pm i \epsilon}(x)=-\int \frac{\mathrm{d}^{4} p}{(2 \pi)^{4}} \frac{m^{2} \mathrm{e}^{-i p \cdot x}}{p^{2}-m^{2} \pm i \epsilon}=\frac{m^{3}}{(2 \pi)^{2}} \frac{K_{1}\left( \pm i m \sqrt{x^{2} \mp i \epsilon}\right)}{\sqrt{x^{2} \mp i \epsilon}}
$$

are the Feynman Green function and its conjugate, $K_{1}$ denoting the modified Bessel function of the second kind. Each $G_{ \pm i \epsilon}(x)$ can be easily evaluated by means of the Wick 
rotation from the Euclidean framework. Then formula (2.4) gives

$$
G_{\mathrm{f}}(x)=\frac{m^{4}}{8 \pi^{2}}\left[\frac{K_{1}\left(i m \sqrt{x^{2}-i \epsilon}\right)}{m \sqrt{x^{2}-i \epsilon}}+\frac{K_{1}\left(-i m \sqrt{x^{2}+i \epsilon}\right)}{m \sqrt{x^{2}+i \epsilon}}\right] .
$$

Observe that $G_{\mathrm{f}}(x-y)$ vanishes for $(x-y)^{2}<0$, so $\phi(x)$ receives contributions only from the past and future light cones

$$
\mathcal{C}^{ \pm}(x) \equiv\left\{y \mid(y-x)^{2} \geqslant 0, \operatorname{sgn}\left(y^{0}-x^{0}\right)= \pm 1\right\}
$$

in $x$, equipped with their interiors. Formula (2.2) can be written as

$$
\langle J\rangle_{\mathrm{f}}(x)=\int_{\mathcal{C}^{-} \cup \mathcal{C}^{+}} G_{\mathrm{f}}(x-y) J(y) \mathrm{d}^{4} y
$$

and the violation of microcausality is due to the contributions of $\mathcal{C}^{+}$.

For $m \sqrt{x^{2}} \gg 1$, we can use the approximation $K_{1}(z) \sim \mathrm{e}^{-z} \sqrt{\pi /(2 z)}$, which holds for $|z| \gg 1, \arg (z) \neq \pi \bmod 2 \pi$. We find the behavior

$$
G_{\mathrm{f}}(x) \sim \frac{m^{5 / 2}}{4 \sqrt{2} \pi^{3 / 2}\left(x^{2}\right)^{3 / 4}} \cos \left(m \sqrt{x^{2}}+\frac{\pi}{4}\right), \quad m \sqrt{x^{2}} \gg 1 .
$$

Since $G_{\mathrm{f}}$ rapidly oscillates for $\sqrt{x^{2}} \gg 1 / m$, only the contributions coming from the regions close to the light cones effectively matter.

In the limit $m \rightarrow \infty, G_{\mathrm{f}}(x)$ is localized in the present, since formula (2.3) gives

$$
G_{\mathrm{f}}(x) \rightarrow \delta^{(4)}(x)
$$

The first terms of the expansion around $m=\infty$, which are

$$
G_{\mathrm{f}}(x)=\left[1-\frac{\square}{m^{2}}+\frac{\square^{2}}{m^{4}}+\cdots\right] \delta^{(4)}(x),
$$

are good indications that, when $m$ is finite, but large, the violation of causality is short range.

However, in the limit $m \rightarrow 0$, using $K_{1}(z) \sim 1 / z$ for $z \rightarrow 0$, we get

$$
G_{\mathrm{f}}(x) \underset{\left|x^{2}\right| \ll 1 / m^{2}}{\longrightarrow} \frac{i m^{2}}{8 \pi^{2}}\left(\frac{1}{x^{2}+i \epsilon}-\frac{1}{x^{2}-i \epsilon}\right)=\frac{m^{2}}{4 \pi} \delta\left(x^{2}\right)
$$

which shows that close enough to the light cones the violation spreads out everywhere with no suppression. 
The question is: is it correct to talk about a violation of microcausality? Or does Lorentz invariance raise it to a violation of macrocausality? Events X and Y separated by the same invariant interval $(x-y)^{2}$ give equal contributions to the fakeon average. If their distance $|\mathbf{x}-\mathbf{y}|$ is small in some inertial frame, it may be arbitrarily large in other inertial frames. Nevertheless, we are going to show that the limit $\left|(x-y)^{2}\right| \ll 1 / m^{2}$ is practically out of reach.

We begin by working in the rest frame of the source. Then we derive a relativistically improved approximate formula. At that point, we will be able to consider the effect of shifting to a moving frame.

We first assume that the source is static and pointlike, i.e.

$$
J(x)=J_{0} \delta^{(3)}(\mathbf{x}-\overline{\mathbf{x}}) .
$$

From the Fourier transform of $G_{\mathrm{f}}$, we easily get the Yukawa behavior

$$
\langle J\rangle_{\mathrm{f}}=\frac{m^{2} \mathrm{e}^{-m r}}{4 \pi r} J_{0}
$$

where $r=|\mathbf{x}-\overline{\mathbf{x}}|$. This result can also be retrieved directly from (2.5) using the tricks explained in the appendix. An extended static source $J(\mathbf{x})$ gives

$$
\langle J\rangle_{\mathrm{f}}(x)=\frac{m^{2}}{4 \pi} \int \mathrm{d}^{3} \mathbf{y} \frac{\mathrm{e}^{-m|\mathbf{x}-\mathbf{y}|}}{|\mathbf{x}-\mathbf{y}|} J(\mathbf{y}) .
$$

The sensitivity of the average to the source is exponentially damped by the distance from the observer.

Next, a pointlike source oscillating with frequency $\omega$,

$$
J\left(x^{0}, \mathbf{x}\right)=J_{0} \mathrm{e}^{-i \omega x^{0}} \delta^{(3)}(\mathbf{x}-\overline{\mathbf{x}})
$$

gives

$$
\langle J\rangle_{\mathrm{f}}(x)=\frac{J_{0} m^{2} \mathrm{e}^{-i \omega x^{0}}}{4 \pi r} \begin{cases}\mathrm{e}^{-r \sqrt{m^{2}-\omega^{2}}} & \text { for } \omega<m, \\ \cos \left(r \sqrt{\omega^{2}-m^{2}}\right) & \text { for } \omega>m .\end{cases}
$$

Note that the fakeon prescription is needed only for $\omega>m$. For $\omega \gg m$ the approximate behavior

$$
\langle J\rangle_{\mathrm{f}}(x) \sim \frac{J_{0} m^{2}}{8 \pi r}\left[\mathrm{e}^{-i \omega\left(x^{0}-r\right)}+\mathrm{e}^{-i \omega\left(x^{0}+r\right)}\right]
$$

shows that the violation of microcausality does propagate along the light cones. 
To treat the most general case, let us introduce the Fourier transform

$$
J\left(x^{0}, \mathbf{x}\right)=\int \frac{\mathrm{d} \omega}{2 \pi} \mathrm{e}^{-i \omega x^{0}} \tilde{J}(\omega, \mathbf{x})
$$

with respect to time. From (2.4) and (2.13), we find

$$
\begin{aligned}
\langle J\rangle_{\mathrm{f}}(x)= & \int_{-m}^{m} \frac{\mathrm{d} \omega}{2 \pi} \mathrm{e}^{-i \omega x^{0}} \int \mathrm{d}^{3} \mathbf{y} \frac{m^{2} \mathrm{e}^{-\sqrt{m^{2}-\omega^{2}}|\mathbf{x}-\mathbf{y}|}}{4 \pi|\mathbf{x}-\mathbf{y}|} \tilde{J}(\omega, \mathbf{y}) \\
& +\int_{|\omega| \geqslant m} \frac{\mathrm{d} \omega}{2 \pi} \mathrm{e}^{-i \omega x^{0}} \int \mathrm{d}^{3} \mathbf{y} \frac{m^{2} \cos \left(\sqrt{\omega^{2}-m^{2}}|\mathbf{x}-\mathbf{y}|\right)}{4 \pi|\mathbf{x}-\mathbf{y}|} \tilde{J}(\omega, \mathbf{y}) .
\end{aligned}
$$

This result shows that all the frequencies $\omega<m$ are exponentially damped by the distance between the observer and the source. Instead, no frequencies $|\omega| \geqslant m$ are damped.

If the source $J\left(y^{0}, \mathbf{y}\right)$ is slowly varying in an amount of time comparable to $1 / m$, i.e.

$$
\tilde{J}(\omega, \mathbf{y})=0 \text { for } \omega>\bar{\omega} \text {, for some } \bar{\omega} \ll m \text {, }
$$

the violation of causality can be estimated by comparing the exact solution

$$
\phi(x)=\langle J\rangle_{\mathrm{f}}(x)=\int_{-\bar{\omega}}^{\bar{\omega}} \frac{\mathrm{d} \omega}{2 \pi} \mathrm{e}^{-i \omega x^{0}} \int \mathrm{d}^{3} \mathbf{y} \frac{m^{2} \mathrm{e}^{-\sqrt{m^{2}-\omega^{2}}|\mathbf{x}-\mathbf{y}|}}{4 \pi|\mathbf{x}-\mathbf{y}|} \tilde{J}(\omega, \mathbf{y})
$$

to the causal formula

$$
\langle J\rangle_{\mathrm{f}}(x) \sim \int \mathrm{d}^{3} \mathbf{y} \frac{m^{2} J\left(x^{0}-|\mathbf{x}-\mathbf{y}|, \mathbf{y}\right)}{4 \pi|\mathbf{x}-\mathbf{y}|} \exp \left(-\sqrt{m^{2}-\bar{\omega}^{2}}|\mathbf{x}-\mathbf{y}|\right) \equiv \tilde{\phi}_{\mathrm{c}}(x),
$$

which involves the source $J$ only at an earlier, retarded time.

To study the accuracy of the causal approximation, let us focus on a pointlike source

$$
J\left(x^{0}, \mathbf{x}\right)=j\left(x^{0}\right) \delta^{(3)}(\mathbf{x}-\overline{\mathbf{x}}),
$$

such that $j\left(x^{0}\right)$ is an $\mathrm{L}^{2}$ function and its Fourier transform $\tilde{j}(\omega)$ vanishes for $\omega>\bar{\omega}$. Then, writing the exact solution $(2.17)$ as $\phi \equiv \tilde{\phi}_{\mathrm{c}}+\Delta \tilde{\phi}$, we have

$$
\mathcal{E} \equiv \frac{\|\Delta \tilde{\phi}\|}{\left\|\tilde{\phi}_{\mathrm{c}}\right\|} \leqslant \frac{\bar{\omega} \sqrt{m+\bar{m}}}{\sqrt{2} \bar{m}^{3 / 2}} \sim \frac{\bar{\omega}}{m}
$$

where $\bar{m} \equiv \sqrt{m^{2}-\bar{\omega}^{2}}$ and $\|\cdots\|$ denotes the $\mathrm{L}^{2}$ norm. This bound gives us a way to estimate the precision of the approximation at the global level. Later we discuss the accuracy of the approximation as a function of the distance $r$ between the source and the observer, to show that the correction $\Delta \tilde{\phi}$, which encodes the violation of microcausality, is negligible for all practical purposes. 


\section{Improved approximation}

The Green function (2.5) is invariant under time reversal T. Instead, the Green function implied by the causal approximation (2.18) is not. A better approximation is given by the T-symmetric formula

$$
\langle J\rangle_{\mathrm{f}}(x) \sim \int \mathrm{d}^{3} \mathbf{y} \frac{m^{2}\left[J\left(x^{0}-|\mathbf{x}-\mathbf{y}|, \mathbf{y}\right)+J\left(x^{0}+|\mathbf{x}-\mathbf{y}|, \mathbf{y}\right)\right]}{8 \pi|\mathbf{x}-\mathbf{y}|} \mathrm{e}^{-\sqrt{m^{2}-\bar{\omega}^{2}}|\mathbf{x}-\mathbf{y}|} \equiv \tilde{\phi}_{\mathrm{T}}(x) .
$$

If we take the source (2.19), with the assumption (2.16), it is easy to check that

$$
\frac{\left\|\tilde{\phi}_{\mathrm{T}}-\tilde{\phi}_{\mathrm{c}}\right\|}{\left\|\tilde{\phi}_{\mathrm{c}}\right\|} \leqslant \frac{\bar{\omega}}{\sqrt{2} \bar{m}} \sim \frac{\bar{\omega}}{\sqrt{2} m} \text {. }
$$

This means that, globally, the T-symmetric approximation is as good as the causal one. Neither (2.17), nor (2.18), nor (2.21) are Lorentz invariant, due to the assumption (2.16). However, (2.21) can be used to switch easily to a moving inertial frame (see below).

\section{Properties}

We list a few properties of the causal approximation (2.18), the T-symmetric approximation (2.21) and the exact solution (2.17).

1) Formula (2.21) has the right behavior for $m \rightarrow 0$,

$$
\lim _{m \rightarrow 0} \frac{\tilde{\phi}_{\mathrm{T}}(x)}{m^{2}}=\frac{1}{8 \pi} \int \frac{\mathrm{d}^{3} \mathbf{y}}{|\mathbf{x}-\mathbf{y}|}\left[J\left(x^{0}-|\mathbf{x}-\mathbf{y}|, \mathbf{y}\right)+J\left(x^{0}+|\mathbf{x}-\mathbf{y}|, \mathbf{y}\right)\right],
$$

in agreement with (2.9), even if, strictly speaking, $m=0$ is not compatible with the

condition of slow variation for time intervals of order $1 / m$. Formula (2.18) does not share this property.

2) When $m$ is large, we correctly get

$$
\lim _{m \rightarrow \infty} \tilde{\phi}_{\mathrm{c}}(x)=\lim _{m \rightarrow \infty} \tilde{\phi}_{\mathrm{T}}(x)=J(x)
$$

in both cases.

3) If $J\left(y^{0}, \mathbf{y}\right)$ has compact support in $\mathbf{y}\left[J\left(y^{0}, \mathbf{y}\right)=0\right.$ for $|\mathbf{y}|>\rho$, where $\rho$ is some finite radius] and the Fourier transform $\tilde{J}(\omega, \mathbf{y})$ vanishes for $\omega>\bar{\omega}, \bar{\omega} \leqslant m$, and is bounded $[|\tilde{J}(\omega, \mathbf{y})| \leqslant K$ for every $\omega$ and $\mathbf{y}]$, then $\phi(x), \tilde{\phi}_{\mathrm{c}}(x)$ and $\tilde{\phi}_{\mathrm{T}}(x)$ tend to zero exponentially at spatial infinity. Indeed, let $|\mathbf{x}|>r$ for a $r>\rho$. Then, $|\mathbf{x}|>r>\rho>|\mathbf{y}|$ implies $|\mathbf{x}-\mathbf{y}|>|\mathbf{x}|-|\mathbf{y}|>r-\rho$, so (2.17), (2.18) and (2.21) give

$$
|\phi|,\left|\tilde{\phi}_{\mathrm{T}}\right|,\left|\tilde{\phi}_{\mathrm{c}}\right|<\frac{m^{2} K \bar{\omega} \rho^{3} \mathrm{e}^{-\bar{m}(r-\rho)}}{3 \pi(r-\rho)} \text {. }
$$




\section{Data and validity of the approximations}

Now we analyse the experimental data to justify the approximations. The theory of quantum gravity that emerges from the fakeon idea predicts a spin- 2 fakeon $\chi_{\mu \nu}$ of mass $m_{\chi}$ and a potential scalar fakeon $\phi$ of mass $m_{\phi}$ (see the next section for details). The masses $m_{\chi}$ and $m_{\phi}$ are free parameters. At present, we do not have strong bounds on their values. For example, the gravitational potential of a point-like mass $M$ is

$$
V(r)=-\frac{G M}{r}\left(1-\frac{4}{3} \mathrm{e}^{-m_{\chi} r}+\frac{1}{3} \mathrm{e}^{-m_{\phi} r}\right) .
$$

Since Newton's law has been verified down to $10^{-2} \mathrm{~cm}[20]$, we infer that

$$
m_{\chi}, m_{\phi} \gtrsim \frac{10^{2}}{\mathrm{~cm}} \sim 10^{-3} \mathrm{eV}
$$

The fakeon masses could have any values larger than this one. If $m_{\chi}, m_{\phi}$ are smaller than the Planck mass, the quantum gravity theory of ref. [1] is perturbative and may allow us to test new physics at relatively low energies. In what follows, we take $m_{\chi}, m_{\phi} \sim 10^{12} \mathrm{GeV}$, which means $1 / m_{\chi}, 1 / m_{\phi} \sim 10^{-36}$ s, as reference values. We also compare the results we obtain with those implied by other values of $m_{\chi}, m_{\phi}$.

The shortest time interval that has been measured directly comes from laser pulses and is about $10^{-17} \mathrm{~s}[21]$. We take this value as the reference amount of time $1 / \bar{\omega}$ when we require that the source is slowly varying at the practical level. If $m_{\chi}, m_{\phi} \sim 10^{12} \mathrm{GeV}$, the overall accuracy (2.20) of the approximation (2.18) is

$$
\mathcal{E}=\frac{\|\Delta \tilde{\phi}\|}{\left\|\tilde{\phi}_{\mathrm{c}}\right\|} \sim \frac{\bar{\omega}}{m} \sim 10^{-19} .
$$

We need $m_{\chi}, m_{\phi} \sim 70 \mathrm{eV}$ to have $\|\Delta \tilde{\phi}\| \sim\left\|\tilde{\phi}_{\mathrm{c}}\right\|$ and make the violation of causality important.

Even in that case, however, we have to fight against the damping exponential factors $\sim \mathrm{e}^{-m r}$ of formulas (2.17), (2.18), (2.21) and (2.24), which depress the outcome. Due to them, the region where the magnitude of $\phi$ is not depressed is a ball of radius $1 / m_{\chi}, 1 / m_{\phi} \sim$ $10^{-26} \mathrm{~cm}$. However, such distances are unreachable to us. Indeed, the shortest distance ever measured, provided by LIGO's technology, is around $10^{-17} \mathrm{~cm}$ [22], where the damping factor is $\sim \mathrm{e}^{-10^{9}}$.

If we want a damping factor of order unity at the shortest distance ever measured, we need $m_{\chi}, m_{\phi} \sim 2 \mathrm{TeV}$. However, in that case $\mathcal{E} \sim 10^{-11}$ is still too small: the causal 
formula (2.18) continues to be good enough and the violation of microcausality remains undetectable.

If the Higgs boson is a fakeon [23], the violation of microcausality extends to an amount of time equal to $1 / m_{H} \sim 10^{-26} \mathrm{~s}$, which is still too short. In that case, $\mathcal{E} \sim 5 \cdot 10^{-10}$ and if we want a damping factor of order one we need to go to distances $r \sim 1 / m_{H} \sim 10^{-16} \mathrm{~cm}$.

Note that, to some extent, it makes sense to assume that the source is slowly varying in time and not assume that it is weakly varying in space, since, as seen, the measurements of space distances are much more precise than those of time intervals. Clearly, the assumption that the source is slowly varying depends on the reference frame and so implies a "spontaneous" breaking of Lorentz symmetry. Can the violation of microcausality be enhanced by switching to a Lorentz frame that moves at a great speed with respect to the source? In principle yes, but in practice no.

To see this, we use the approximation (2.21). Let $s$ denote the frame where the assumption (2.16) holds. If we go to a Lorentz frame $s^{\prime}$ that moves at a speed $\beta>0$ relatively to the source $J$ in $s$, the frequencies of $J$ are enhanced by factors

$$
\sqrt{\frac{1-\beta}{1+\beta}}, \quad \sqrt{\frac{1+\beta}{1-\beta}} .
$$

If we switch to an inertial frame with $\beta=1-\varepsilon, \varepsilon \ll 1$, the larger factor (2.27) is $\sim \sqrt{2 / \varepsilon}$. If the maximal frequency of the source $J$ is $\bar{\omega}^{\prime}$, as seen from $s^{\prime}$, the maximal frequency seen from $s$ is $\bar{\omega} \sim \bar{\omega}^{\prime} \sqrt{2 / \varepsilon}$. Then, if we assume $m_{\chi}, m_{\phi} \sim 10^{12} \mathrm{GeV}$ and take $1 / \bar{\omega}^{\prime}$ around the shortest amount of time that has been measured directly so far, which means $\bar{\omega}^{\prime} \sim 10^{17} \mathrm{~Hz}$, we need $\varepsilon \sim 10^{-38}$ to make the right-hand sides of (2.20) and (2.22) of order one, which is out of reach. Similar conclusions hold with the other values of $m_{\chi}, m_{\phi}$ considered above and for the transverse Doppler effect.

The second question is: can we reduce the effects of the damping factor $\mathrm{e}^{-m r}$ ? The answer is, again, no, because measurements of space distances mix with measurements of time intervals, which are much less precise. Consider the contraction of lengths in special relativity. The endpoints of a bar of length $l$ at rest in $s$ must be recorded simultaneously in $s^{\prime}$. However, if the highest precision of a measurement of time in $s^{\prime}$ is $\Delta t^{\prime} \sim 10^{-17} \mathrm{~s}$, then the length

$$
l^{\prime}=\frac{l}{\gamma} \pm|\beta| \Delta t^{\prime}
$$

of the bar in $s^{\prime}$ has an error that cannot be reduced below $\sim 10^{-7} \mathrm{~cm}$ for $|\beta| \rightarrow 1$. On thop of this, the factor $\gamma$ is practically one for all known macroscopic objects of our galaxy (and 
far beyond), which have maximum velocities of order $1000 \mathrm{~km} / \mathrm{s}$ with respect to the CMB rest frame. Large values of $\gamma$ are hard to reach even for elementary particles, because we would need to accelerate them to about $10^{12} \mathrm{GeV}$.

In conclusion, there is no obvious way of reducing the shortest time intervals or space distances we can measure by switching to different Lorentz frames. When the source is slowly varying in time the fakeon average is short range. If, in addition, $J$ has compact support in space, $\langle J\rangle_{\mathrm{f}}$ is exponentially decreasing at spatial distances. Under these circumstances, the violation of microcausality is a sort of fuzziness of the source and its evolution.

A last resort to amplify the violations is to get help from radiation, i.e. signals that do propagate along the light cones. The electromagnetic radiation is not very helpful here, since only gravity is sensitive to the averaged source $\langle J\rangle_{\mathrm{f}}$, while the other interactions are sensitive to the unaveraged source $J$. In the next section we show that the gravitational radiation predicted by the classicization of quantum gravity coincides with the one predicted by the Einstein theory.

\section{Gravitational waves}

Now we study the effects on the gravitational waves. We assume that the sources are smooth, have compact support in space and are slowly varying for time intervals of order $1 / m$, where $m$ is the fakeon mass.

We recall that the quantum gravity theory of ref. [1] describes a triplet made of the graviton, a spin- 2 fakeon $\chi_{\mu \nu}$ of mass $m_{\chi}$ and a scalar field $\phi$ of mass $m_{\phi}$, which can be fake or physical. This leads to two physically inequivalent theories, the GFF (gravitonfakeon-fakeon) theory and the GSF (graviton-scalar-fakeon) theory. In this section we show that the gravitational waves predicted by both versions coincide with the ones of Einstein gravity for all practical purposes. For definiteness, we first work in the GFF theory and then extend the results to the GSF theory.

Neglecting the cosmological constant, the unprojected field equations that follow from the interim classical action are [19]

$$
\left(1+\frac{\nabla^{2}}{m_{\chi}^{2}}\right) G_{\mu \nu}+\frac{r_{\phi \chi}}{3}\left(\nabla_{\mu} \nabla_{\nu}-g_{\mu \nu} \nabla^{2}\right) G=\kappa^{2} T_{\mu \nu}
$$


where $G_{\mu \nu}$ is the Einstein tensor, $r_{\phi \chi}=\left(m_{\phi}^{2}-m_{\chi}^{2}\right) /\left(m_{\phi}^{2} m_{\chi}^{2}\right), \kappa=\sqrt{8 \pi G}$ and

$$
\kappa^{2} T_{\mu \nu} \equiv \kappa^{2} T_{\mathfrak{m} \mu \nu}+\frac{1}{2 m_{\chi}^{2}} g_{\mu \nu} R^{\rho \sigma} R_{\rho \sigma}-\frac{2}{m_{\chi}^{2}} R_{\mu \rho \nu \sigma} R^{\rho \sigma}+\frac{2 m_{\phi}^{2}+m_{\chi}^{2}}{3 m_{\chi}^{2} m_{\phi}^{2}} R\left(R_{\mu \nu}-\frac{1}{4} g_{\mu \nu} R\right)
$$

$T_{\mathfrak{m} \mu \nu}$ denoting the matter energy-momentum tensor.

Once the fakeons $\phi$ and $\chi_{\mu \nu}$ are projected away, the field equations for the graviton field $h_{\mu \nu}$, defined as the fluctuation around flat space by means of the relation $g_{\mu \nu}=\eta_{\mu \nu}+2 \kappa h_{\mu \nu}$, have the form:

$$
G_{\mu \nu}=\kappa^{2} T_{\mu \nu}^{\mathrm{GFF}}
$$

where $T_{\mu \nu}^{\mathrm{GFF}}$ contains the corrections to the Einstein equations. Since the right-hand side depends on the metric, the equations have to be treated perturbatively in $\kappa$. We expand the Einstein tensor as

$$
G_{\mu \nu}=\kappa\left[-\square \tilde{h}_{\mu \nu}+\partial_{\mu} \partial^{\rho} \tilde{h}_{\rho \nu}+\partial_{\nu} \partial^{\rho} \tilde{h}_{\rho \mu}-\eta_{\mu \nu} \partial^{\rho} \partial^{\sigma} \tilde{h}_{\rho \sigma}\right]+\kappa^{2} J_{\mu \nu}
$$

where

$$
\tilde{h}_{\mu \nu}=h_{\mu \nu}-\frac{1}{2} \eta_{\mu \nu} h, \quad h=h_{\mu \nu} \eta^{\mu \nu},
$$

$\square=\partial^{2}$ and $J_{\mu \nu}$ is at least quadratic in $h_{\alpha \beta}$. Using the definition of $T_{\mu \nu}^{\text {GFF }}$ given in ref. [19], equation (3.3) can be recast in the perturbative form

$-\square \tilde{h}_{\mu \nu}+\partial_{\mu} \partial^{\rho} \tilde{h}_{\rho \nu}+\partial_{\nu} \partial^{\rho} \tilde{h}_{\rho \mu}-\eta_{\mu \nu} \partial^{\rho} \partial^{\sigma} \tilde{h}_{\rho \sigma}=\kappa\left\langle T_{\mu \nu}-U_{\mu \nu}+\frac{r_{\phi \chi}}{3}\left(\eta_{\mu \nu} \partial^{2}-\partial_{\mu} \partial_{\nu}\right)\langle T-U\rangle_{\phi}\right\rangle_{\chi}$,

where $\kappa^{2} U_{\mu \nu}$ is the left-hand side of (3.1) minus its linear part, $T=\eta^{\mu \nu} T_{\mu \nu}, U=\eta^{\mu \nu} U_{\mu \nu}$ and the fakeon average $\langle\cdots\rangle_{\mathrm{f}}$ is defined as in formula $(2.3)$, the masses being $m^{2}=m_{\chi}^{2}$ or $m^{2}=m_{\phi}^{2}$, depending on the case.

It is convenient to impose the gauge-fixing condition $\partial^{\mu} \tilde{h}_{\mu \nu}=\partial_{\nu} V$, where $V$ is a function to be determined, because then the equations (3.4) take the form

$$
\square\left(\tilde{h}_{\mu \nu}-\eta_{\mu \nu} V\right)=-\kappa\left\langle T_{\mu \nu}-U_{\mu \nu}\right\rangle_{\chi}+\left(\partial_{\mu} \partial_{\nu}-\eta_{\mu \nu} \square\right)\left[2 V+\frac{\kappa r_{\phi \chi}}{3}\left\langle\langle T-U\rangle_{\phi}\right\rangle_{\chi}\right]
$$

and if we choose

$$
V=-\frac{\kappa r_{\phi \chi}}{6}\left\langle\langle T-U\rangle_{\phi}\right\rangle_{\chi}
$$

they reduce to

$$
\square\left(\tilde{h}_{\mu \nu}+\frac{\kappa \eta_{\mu \nu} r_{\phi \chi}}{6}\left\langle\langle T-U\rangle_{\phi}\right\rangle_{\chi}\right)=-\kappa\left\langle T_{\mu \nu}-U_{\mu \nu}\right\rangle_{\chi}
$$


Applying the definition (2.3) in momentum space, it is easy to prove the identity

$$
r_{\phi \chi}\left\langle\langle T-U\rangle_{\phi}\right\rangle_{\chi}=\frac{1}{m_{\chi}^{2}}\langle T-U\rangle_{\chi}-\frac{1}{m_{\phi}^{2}}\langle T-U\rangle_{\phi}
$$

Inserting this result into (3.6), we obtain the equation

$$
\square\left(\tilde{h}_{\mu \nu}+\frac{\kappa \eta_{\mu \nu}}{6 m_{\chi}^{2}}\langle T-U\rangle_{\chi}-\frac{\kappa \eta_{\mu \nu}}{6 m_{\phi}^{2}}\langle T-U\rangle_{\phi}\right)=-\kappa\left\langle T_{\mu \nu}-U_{\mu \nu}\right\rangle_{\chi} .
$$

We concentrate on the first order in $\kappa$, where $U_{\mu \nu}=J_{\mu \nu}=0$. Since (3.7) implies $\tilde{h}_{\mu \nu}=\mathcal{O}(\kappa)$, we have $R^{2} \sim R_{\mu \nu} R^{\mu \nu} \sim \kappa^{4}$. Then, formula (3.2) implies $T_{\mu \nu}=T_{\mathfrak{m} \mu \nu}+\mathcal{O}\left(\kappa^{2}\right)$. At the end, the approximation leads to the equation

$$
\square\left(\tilde{h}_{\mu \nu}+\frac{\kappa \eta_{\mu \nu}}{6 m_{\chi}^{2}}\left\langle T_{\mathfrak{m}}\right\rangle_{\chi}-\frac{\kappa \eta_{\mu \nu}}{6 m_{\phi}^{2}}\left\langle T_{\mathfrak{m}}\right\rangle_{\phi}\right)=-\kappa\left\langle T_{\mathfrak{m} \mu \nu}\right\rangle_{\chi}
$$

It is convenient to decompose

$$
\tilde{h}_{\mu \nu}=\tilde{h}_{\mu \nu}^{E}+\tilde{h}_{\mu \nu}^{\mathrm{f}}
$$

as the sum of the solution

$$
\tilde{h}_{\mu \nu}^{E}\left(x^{0}, \mathbf{x}\right)=-\frac{\kappa}{4 \pi} \int \mathrm{d}^{3} \mathbf{y} \frac{T_{\mathfrak{m} \mu \nu}\left(x^{0}-|\mathbf{x}-\mathbf{y}|, \mathbf{y}\right)}{|\mathbf{x}-\mathbf{y}|}
$$

to the Einstein equations

$$
\square \tilde{h}_{\mu \nu}=-\kappa T_{\mathfrak{m} \mu \nu}
$$

(in the same approximation) and the rest $\tilde{h}_{\mu \nu}^{\mathrm{f}}$, which is due to the fakeons. Combining (3.8) and (3.9), it is easy to find that the difference $\tilde{h}_{\mu \nu}^{\mathrm{f}}$ solves the equation

$$
\square\left(\tilde{h}_{\mu \nu}^{\mathrm{f}}+\frac{\kappa \eta_{\mu \nu}}{6 m_{\chi}^{2}}\left\langle T_{\mathfrak{m}}\right\rangle_{\chi}-\frac{\kappa \eta_{\mu \nu}}{6 m_{\phi}^{2}}\left\langle T_{\mathfrak{m}}\right\rangle_{\phi}\right)=\kappa\left(T_{\mathfrak{m} \mu \nu}-\left\langle T_{\mathfrak{m} \mu \nu}\right\rangle_{\chi}\right)=\kappa \frac{\square}{m_{\chi}^{2}}\left\langle T_{\mathfrak{m} \mu \nu}\right\rangle_{\chi} .
$$

In the end, the solution reads

$$
\tilde{h}_{\mu \nu}^{\mathrm{f}}=\frac{\kappa}{m_{\chi}^{2}}\left\langle T_{\mathfrak{m} \mu \nu}\right\rangle_{\chi}-\frac{\kappa \eta_{\mu \nu}}{6 m_{\chi}^{2}}\left\langle T_{\mathfrak{m}}\right\rangle_{\chi}+\frac{\kappa \eta_{\mu \nu}}{6 m_{\phi}^{2}}\left\langle T_{\mathfrak{m}}\right\rangle_{\phi} .
$$

We see that $\tilde{h}_{\mu \nu}^{\mathrm{f}}$ is a sum of fakeon averages, which obey the properties derived in the previous section. Therefore, if the source is slowly varying the corrections are short-range 
and do not affect the radiation, which coincides with the one predicted by the Einstein equations. If the source is not slowly varying, we also have contributions such as those appearing in the second line of formula (2.13) with $\omega>m=m_{\phi}, m_{\chi}$.

In the GSF theory, where $\phi$ is a physical particle, only $\chi_{\mu \nu}$ is a fakeon, so the fakeon average $\langle\cdots\rangle_{\phi}$ that appears in the solution (3.11) is replaced by the convolution $\langle\cdots\rangle_{\phi r e t}$ with the retarded Yukawa potential. We obtain

$$
\tilde{h}_{\mu \nu}^{\mathrm{GSF}}=\tilde{h}_{\mu \nu}^{E}+\frac{\kappa}{m_{\chi}^{2}}\left\langle T_{\mathfrak{m} \mu \nu}\right\rangle_{\chi}-\frac{\kappa \eta_{\mu \nu}}{6 m_{\chi}^{2}}\left\langle T_{\mathfrak{m}}\right\rangle_{\chi}+\frac{\kappa \eta_{\mu \nu}}{6 m_{\phi}^{2}}\left\langle T_{\mathfrak{m}}\right\rangle_{\phi \mathrm{ret}}
$$

In the end, the gravitational waves do not amplify or propagate the violation of microcausality, due to the large distances involved and the damping exponential factors that appear in formulas (2.17), (2.18), (2.21) and (2.24). The bounds on the masses $m_{\chi}$ and $m_{\phi}$ that we can obtain from this analysis are much less meaningful than the bound (2.25) obtained from the Newton force.

The conclusion holds under the assumption (2.16) that the sources are slowly varying in time, which means that their frequencies are much smaller than the fakeon masses $m_{\chi}$ and $m_{\phi}$. As shown by the second line of formula (2.15), frequencies of order $m_{\chi}, m_{\phi}$ or higher are not damped. Core-collapse supernovae are expected to generate waves with frequencies up to about $10 \mathrm{kHz}[24]$. With fakeon masses of the order of $10^{12} \mathrm{GeV}$, those frequencies are still too small, but they become important if $m_{\chi}, m_{\phi}$ are around one $\mathrm{MeV}$, which we cannot exclude, yet.

We have mentioned that the effects of the fakeon average are a microuncertainty on the source of the radiation. One might wonder why such an uncertainty does not amplify, in the end. The answer is that all the gravitational signals emitted by the averaged source propagate with the same speed $c$, which implies that, at arbitrary distances, the microuncertainty is just translated in spacetime along the light cones, but not amplified.

\section{Hubble constant and recovery of microcausality}

In this section we study other situations where the potential violation of microcausality is depressed rather than enhanced. In passing, the investigation gives us the opportunity to illustrate some important aspects of the classical limit of the fakeon prescription.

We consider a scalar field $\varphi$ (different from the scalar $\phi$ belonging to the graviton triplet) in the Friedmann-Lemaitre-Robertson-Walker (FLRW) background. Before plunging into the details, it is important to make a few comments on the properties of our theory 
on nontrivial backgrounds. The spin-2 fakeon $\chi_{\mu \nu}$ is described by an involved action [10], whose quadratic part is the covariantized Pauli-Fierz action [25], plus nonminimal terms. It is known that a Pauli-Fierz mass term can create pathologies [26] on nontrivial backgrounds. For example, it may turn on ghost-like degrees of freedom. Nevertheless, these problems do not arise in the theory of quantum gravity we are studying. Recall that the Pauli-Fierz action of $\chi_{\mu \nu}$ has the wrong overall sign (which is why $\chi_{\mu \nu}$ is treated as a fakeon). This means that any degree of freedom turned on by its mass term would have the right sign and be healthy. Actually, that degree of freedom is already present in the theory (and under control): it is the massive scalar $\phi$.

To see this, one must recall how the fields $\phi$ and $\chi_{\mu \nu}$ are introduced [10]. One starts from the higher-derivative action

$$
S_{\mathrm{QG}}=-\frac{M_{\mathrm{Pl}}^{2}}{16 \pi} \int \mathrm{d}^{4} x \sqrt{-g}\left[R+\frac{1}{m_{\chi}^{2}}\left(R_{\mu \nu} R^{\mu \nu}-\frac{1}{3} R^{2}\right)-\frac{1}{6 m_{\phi}^{2}} R^{2}\right]
$$

(neglecting the cosmological term, for simplicity) and introduces auxiliary fields to convert it into a two-derivative action. The scalar $\phi$ is originated by the auxiliary field for $R^{2}$, while the tensor $\chi_{\mu \nu}$ is originated by the auxiliary field for $R_{\mu \nu} R^{\mu \nu}-R^{2} / 3$. This means that the trace of $\chi_{\mu \nu}$ is not really an independent field (it can also be seen as originated by an auxiliary field for $R^{2}$ ), so it talks with $\phi$. Thus, a degree of freedom turned on by the $\chi_{\mu \nu}$ Pauli-Fierz mass term is not independent, but can be reabsorbed into $\phi$.

Other situations that lead to healthy massive Pauli-Fierz fields are known in the literature, as in the de Rham-Gabadadze-Tolley model [27] or the compactification of fivedimensional theories [28].

We also stress that the concept of fakeon is more general than the theory of quantum gravity it comes from. One can apply it to models of quantum gravity that do not include massive spin-2 fields (renouncing renormalizability, as in Einstein gravity), but also theories of matter fields (and fakeons) in curved space. As already recalled, even the Higgs boson might be a fakeon [23]. In this sense, the results of this section contribute to the analysis of the general properties of fakeons in connection with the issue of microcausality.

Coming back to the problem of this section, the equation of a generic scalar $\varphi$ of mass $m$ interacting with an external source $J$ in a curved background is

$$
\frac{1}{\sqrt{-g}} \partial_{\mu}\left(\sqrt{-g} g^{\mu \nu} \partial_{\nu} \varphi\right)+m^{2} \varphi=J
$$

We study it under the assumption of homogeneity, $\varphi=\varphi(t)$, in the FLRW background. 
The equation then reads

$$
\Sigma \varphi=\frac{J}{m^{2}}
$$

where $\Sigma$ denotes the operator

$$
\Sigma=1+\frac{3 H}{m^{2}} \frac{\mathrm{d}}{\mathrm{d} t}+\frac{1}{m^{2}} \frac{\mathrm{d}^{2}}{\mathrm{~d} t^{2}}
$$

$H=\dot{a}(t) / a(t)$ is the Hubble parameter and $a(t)$ is the cosmic scale factor. The Green function $G_{H}(t)$ is the solution of

$$
\Sigma G_{H}(t)=\delta(t)
$$

If $\varphi$ is a fakeon, the solution is

$$
\varphi(t)=\int_{-\infty}^{+\infty} \mathrm{d} t^{\prime} G_{H}^{\mathrm{f}}\left(t-t^{\prime}\right) J\left(t^{\prime}\right) \equiv \frac{1}{m^{2}}\langle J\rangle_{\Sigma}(t),
$$

where the fakeon average is defined as

$$
\langle A\rangle_{X} \equiv \frac{1}{2}\left[\left.\frac{1}{X}\right|_{\mathrm{rit}}+\left.\frac{1}{X}\right|_{\mathrm{adv}}\right] A .
$$

Let us recall that the fakeon prescription is originated perturbatively, in momentum space (see e.g. [19]). For this reason, it is convenient to study the Fourier transforms $\tilde{G}_{H}(\omega), \tilde{G}_{H}^{\mathrm{f}}(\omega)$ of $G_{H}(t)$ and $G_{H}^{\mathrm{f}}(t)$. The retarded and advanced potentials are defined by shifting the frequency $\omega$ to $\omega \pm i \epsilon$ and taking the anti-Fourier transforms.

In the limit $H \rightarrow 0$ we find

$$
\Sigma \rightarrow 1+\frac{1}{m^{2}} \frac{\mathrm{d}^{2}}{\mathrm{~d} t^{2}}
$$

which is the one-dimensional version of the operator studied in section 2 and gives the fakeon Green function [19]

$$
G_{0}^{\mathrm{f}}(t)=\frac{m}{2} \sin (m|t|) .
$$

Here the violation of microcausality is generically negligible due to the rapidly oscillating behavior. At the cosmological level, on the other hand, short time intervals can be important in the first moments of the universe, so it is interesting to study the problem at nonzero $H$.

A situation that we can investigate exactly is the case of the vacuum energy, where $H$ is constant. Since we can at most assume $H \sim$ constant for a finite amount of time, we study the equation (4.2) in some interval

$$
t_{1} \leqslant t \leqslant t_{2}
$$


We compare the cases where $\varphi$ is physical and $\varphi$ is fake. The most general solution for the Fourier transform $\tilde{G}_{H}(\omega)$ is

$$
\tilde{G}_{H}(\omega)=-\frac{m^{2}}{\left(\omega-\omega_{+}\right)\left(\omega-\omega_{-}\right)}+A(2 \pi) \delta\left(\omega-\omega_{+}\right)+B(2 \pi) \delta\left(\omega-\omega_{-}\right),
$$

where $A$ and $B$ are arbitrary constants,

$$
\omega_{ \pm}=-\frac{3}{2} H i \pm \sigma, \quad \sigma=\sqrt{m^{2}-\frac{9}{4} H^{2}}
$$

and the "complex delta function $\delta$ " has to be understood as a series expansion in powers of $H / m$. Note that at $H \neq 0$ the nonvanishing imaginary parts of $\omega_{ \pm}$make the prescriptions $\omega \rightarrow \omega \pm i \epsilon$ redundant.

If $\varphi$ is a physical field, $A$ and $B$ are determined by the initial conditions. Instead, if $\varphi$ is a fakeon, we must set $A$ and $B$ to zero, since the "on-shell" contributions $\delta\left(\omega-\omega_{ \pm}\right)$ must be absent, by definition. Thus, the fakeon Green function turns out to be

$$
G_{H}^{\mathrm{f}}(t)=-\int \frac{\mathrm{d} \omega}{2 \pi} \frac{m^{2} \mathrm{e}^{-i \omega t}}{\left(\omega-\omega_{+}\right)\left(\omega-\omega_{-}\right)}=m^{2} \operatorname{sgn}(H) \theta(H t) \mathrm{e}^{-\frac{3}{2} H t} \frac{\sin (t \sigma)}{\sigma},
$$

which we have written in a form that is explicit for both real and imaginary $\sigma$. Due to the theta function of (4.8), when $H$ is positive only the past contributes to the fakeon solution

$$
\varphi(t)=\int_{-\infty}^{t} \mathrm{~d} t^{\prime} G_{H}^{\mathrm{f}}\left(t-t^{\prime}\right) J\left(t^{\prime}\right)
$$

which means that the violation of microcausality disappears altogether. It does not matter whether $m$ is small or large, since the result is exact. If, on the other hand, $H$ is negative the opposite occurs.

The projection drops the delta-function contributions of formula (4.7). Note that it is not straightforward to make the projection directly in coordinate space, because the differential equation is only defined in the interval (4.6). For example, we cannot discard alleged "runaway solutions". Moreover, the expressions of $\omega_{ \pm}$show that for $H>0$ the runaway behavior concerns $t \rightarrow-\infty$, which makes no sense if the universe has a beginning. Not to mention that the unknown differential equation for $t<t_{1}$ could make the runaway behavior disappear.

Yet, formula (4.9) hides a subtlety: it requires knowledge of the source $J$ for $t<t_{1}$. We might have that knowledge or not [we just know that the differential equation is (4.2) 
with $H=$ constant for $t_{1} \leqslant t \leqslant t_{2}$ ]. What if we do not know $J$ in the far past (e.g. if the universe has a beginning)?

To clarify this point, it is useful to consider the case where $\varphi$ is a physical field, where the most general solution can be written as

$$
\varphi(t)=\int_{t_{1}}^{t_{2}} \mathrm{~d} t^{\prime} G_{H}^{\mathrm{f}}\left(t-t^{\prime}\right) J\left(t^{\prime}\right)+\mathrm{e}^{-\frac{3}{2} H t}\left[A^{\prime} \cos (\sigma t)+\frac{B^{\prime}}{\sigma} \sin (\sigma t)\right],
$$

where $A^{\prime}$ and $B^{\prime}$ are new constants. Here the problem of knowing $J$ at times prior to $t_{1}$ does not show up, since this knowledge is hidden into $A^{\prime}$ and $B^{\prime}$. However, when $\varphi$ is a fakeon we do not have such constants and the problem remains.

What saves the day is that the damping factor and the oscillating behavior of $G_{H}^{\mathrm{f}}$ restrict the relevant contributions of the integral (4.9) to a little bit of future and a little bit of past around $t$. Let $\Delta t=\min (2 /(3|H|), 1 / \sigma)$ for $m>3 H / 2$ and $\Delta t=\max \left(1 /\left|\omega_{+}\right|, 1 /\left|\omega_{-}\right|\right)$ for $m<3 H / 2$. If $J$ is regular and tends to zero at infinity, the survining uncertainty

$$
\delta J \equiv \int_{-\infty}^{t_{1}} \mathrm{~d} t^{\prime} G_{H}^{\mathrm{f}}\left(t-t^{\prime}\right) J\left(t^{\prime}\right)
$$

is small for all times $t \gtrsim t_{1}+\Delta t$. Thus, we can replace (4.9) with the approximate solution

$$
\varphi(t)=\int_{t_{1}}^{t} \mathrm{~d} t^{\prime} G_{H}^{\mathrm{f}}\left(t-t^{\prime}\right) J\left(t^{\prime}\right) \quad \text { for } t_{1}+\Delta t \lesssim t \leqslant t_{2} .
$$

This result shows that we get predictivity in an interval that is slightly smaller than (4.9).

In the end, we learn that when fakeons are present the differential equations must be understood in a new way. In particular, we may have to deal with uncertainties and fuzziness every time we use them.

When $H$ is approximately constant, we obtain an approximate solution by replacing $H$ with $H(t)$. In ref. [18] it was shown that the equations of the FLRW metric for the GFF theory coincide with the Friedmann equations upon making the replacements $\rho-3 p \rightarrow\langle\rho-3 p\rangle_{\Sigma}$ and $\rho+p \rightarrow\langle\rho+p\rangle_{\Upsilon}$. The mass appearing in $\Sigma$ is $m_{\phi}$ and $\Upsilon=\Sigma+6 \dot{H} / m_{\phi}^{2}$ (at zero space curvature). Then the result (4.9) implies that when $H$ is constant and positive, as in the primordial, inflationary phase of the universe, microcausality is restored in all the equations of the GFF theory. In some sense, the positivity of the Hubble constant determines the direction of time in the early universe.

After inflation, $H$ remains positive, but not constant. We do not have the general solution $G_{H}^{\mathrm{f}}(t)$ for a generic function $H(t)$. Nevertheless, if $H \lesssim m_{\phi}$ we can neglect the 
time dependence of $H$ for intervals of time $\delta t$ much smaller than the Hubble time $t_{H}=1 / H$. Indeed, the usual Friedmann equations imply $|\dot{H}| \lesssim H^{2}$ for $p=w \rho$. If $H \lesssim m_{\phi}$, we have $\Sigma \sim \Upsilon$ and the inequality $|\dot{H}| \lesssim H^{2}$ is also implied by the GFF equations that follow from the classicization of quantum gravity. Combining $|\dot{H}| \lesssim H^{2}$ with $|\delta t| \ll t_{H}$, we obtain $|\delta t| \ll H /|\dot{H}|$, which means $H(t) \sim H=$ constant. Then we can repeat the arguments outlined above and reach similar conclusions. This means that for amounts of time much smaller than the Hubble time (which is comparable to the life of the universe), there is no violation of microcausality in the classical limit.

Finally, note that the limits

$$
G_{0^{ \pm}}^{\mathrm{f}}(t) \equiv \lim _{H \rightarrow 0^{ \pm}} G_{H}^{\mathrm{f}}(t)=m \theta( \pm t) \sin (m|t|)
$$

do not coincide with the $H=0$ Green function $G_{0}^{\mathrm{f}}(t)$ of formula $(4.5)$. Actually, $G_{0}^{\mathrm{f}}(t)=$ $\left(G_{0^{+}}^{\mathrm{f}}(t)+G_{0^{-}}^{\mathrm{f}}(t)\right) / 2$. Basically, the resummation of the expansion in powers of $H$ acts as a bifurcation.

\section{Conclusions}

The results of the investigations carried out in this paper are good news for the consistency of the theory of quantum gravity of ref. [1] with data. At the same time, they mean that more efforts have to be spent to identify ways to test the first departures from the predictions of Einstein gravity.

The violation of microcausality is expressed by a fuzziness relation

$$
\Delta x^{2} \sim \frac{1}{m^{2}}
$$

where $m$ is the fakeon mass and $\Delta x$ is the invariant interval between two events. The relation (5.1) means that events separated by an interval $\Delta x$ of order $1 / m$ cannot be chronologically ordered or distinguished from each other. Because of the damping factor $\mathrm{e}^{-m r}$ of formulas $(2.17),(2.18),(2.21)$ and $(2.24)$, under normal circumstances (5.1) basically means $|\Delta t| \sim 1 / m$, i.e. time does not make sense below the Compton wavelength of the fakeon. Although the relation $|\Delta t| \sim 1 / m$ is not Lorentz invariant, the apparent breakdown of Lorentz symmetry is of a spontaneous type, due to the limitations of our experimental accuracies. The measurements of time intervals are much less precise than those of space distances. Moreover, we cannot change inertial frame at will. Actually, the 
subset of inertial frames spanned by the macroscopic objects populating our galaxy and far beyond is rather tiny. This makes our perception of the world quite limited, if not biased. The idea of microcausality we inherit from it might just be a blunder suggested by our partial insight and experimental inaccuracy.

Not to mention that in several situations, the violation of microcausality disappears altogether, for a variety of reasons. For example, the positivity of the Hubble constant makes the fakeon average causal and is ultimately responsible for the arrow of time in the early universe.

\section{Acknowledgments}

We are grateful to M. Porrati for helpful discussions.

\section{A Appendix}

It is interesting to prove the limit (2.8) directly in Minkowski spacetime, to point out some nontrivial aspects of the fakeon Green function and describe how the light cone contributions (2.9) disappear. Consider

$$
\int \mathrm{d}^{4} x G_{\mathrm{f}}(x) J(x)
$$

where $J(x)$ denotes a test function. If we rescale $x \rightarrow x / m$, we obtain $J(x / m)$ (which tends to $J(0)$ for $m \rightarrow \infty$ and can be taken outside the integral) times an $m$-independent integral. The latter must be computed with the help of a cutoff and a trick to properly account for the light-cone contributions (2.9).

We switch to polar coordinates $(t, r, \theta, \varphi)$, insert the cutoff $L$ on the $r$ integral (for $r$ large) and integrate the angles away. Then we separate the integral into the sum of three contributions, to isolate the light cones from the rest: $(i)$ the integral for $\left|x^{2}\right| \leqslant \delta^{2} ;(i i)$ the integral for $x^{2} \geqslant \delta^{2}$ and (iii) the integral for $x^{2} \leqslant-\delta^{2}$, with $\delta$ arbitrarily small. In $(i)$ we use the approximation (2.9) and obtain

$$
2 J(0) \int_{0}^{L} r^{2} \mathrm{~d} r \int_{-\theta\left(r^{2}-\delta^{2}\right) \sqrt{r^{2}-\delta^{2}}}^{\sqrt{r^{2}+\delta^{2}}} \mathrm{~d} t \delta\left(t^{2}-r^{2}\right)=J(0) \frac{L^{2}}{2} .
$$

In $(i i)$ we get

$$
\frac{J(0)}{\pi} \int_{0}^{L} r^{2} \mathrm{~d} r \int_{0}^{+\infty} \mathrm{d} s \frac{K_{1}(i s)+K_{1}(-i s)}{\sqrt{s^{2}+r^{2}}}=J(0)\left[1-\frac{L^{2}}{2}-\mathrm{e}^{-L}(L+1)\right] .
$$


We have simplified this expression by switching to the variables $s, r$, where $s=\sqrt{t^{2}-r^{2}}$ and noting that if we take $\epsilon \rightarrow 0$, the integrand turns out to be regular for $s=0$. In particular, we can let $\delta \rightarrow 0$ here, i.e. integrate $s$ from 0 to infinity. Finally, the integral (iii) vanishes, since $G_{\mathrm{f}}(x)=0$ for $x^{2}<0$. Summing (6.1) and (6.2) and taking $L \rightarrow \infty$, we get $J(0)$, as we had to prove.

The nontrivial point is that the contributions (6.1) from the light cones (2.9) are divergent, but so are the bulk contributions (6.2) and the total is finite.

\section{References}

[1] D. Anselmi, On the quantum field theory of the gravitational interactions, J. High Energy Phys. 06 (2017) 086, 17A3 Renormalization.com and arXiv: 1704.07728 [hep-th].

[2] D. Anselmi, Fakeons and Lee-Wick models, J. High Energy Phys. 02 (2018) 141, 18A1 Renormalization.com and arXiv:1801.00915 [hep-th].

[3] T.D. Lee and G.C. Wick, Negative metric and the unitarity of the S-matrix, Nucl. Phys. B 9 (1969) 209.

[4] T.D. Lee and G.C. Wick, Finite theory of quantum electrodynamics, Phys. Rev. D 2 (1970) 1033.

[5] R.E. Cutkosky, P.V Landshoff, D.I. Olive and J.C. Polkinghorne, A non-analytic S matrix, Nucl. Phys. B12 (1969) 281.

[6] N. Nakanishi, Lorentz noninvariance of the complex-ghost relativistic field theory, Phys. Rev. D 3, 811 (1971).

[7] D. Anselmi and M. Piva, A new formulation of Lee-Wick quantum field theory, J. High Energy Phys. 06 (2017) 066, 17A1 Renormalization.com and arXiv:1703.04584 [hep-th];

D. Anselmi and M. Piva, Perturbative unitarity in Lee-Wick quantum field theory, Phys. Rev. D 96 (2017) 045009 and 17A2 Renormalization.com and arXiv:1703.05563 [hep-th]. 
[8] D. Anselmi, Fakeons, unitarity, massive gravitons and the cosmological constant, J. High Energy Phys. 12 (2019) 027, 19A2 Renormalization.com and arXiv:1909.04955 [hep-th].

[9] D. Anselmi and M. Piva, The ultraviolet behavior of quantum gravity, J. High Energ. Phys. 05 (2018) 27, 18A2 Renormalization.com and arXiv:1803.07777 [hep-th].

[10] D. Anselmi and M. Piva, Quantum gravity, fakeons and microcausality, J. High Energy Phys. 11 (2018) 21, 18A3 Renormalization.com and arXiv:1806.03605 [hep-th].

[11] J.D. Jackson, Classical electrodynamics, John Wiley and Sons, Inc. (1975), chap. 17.

[12] L. Bel and H. Sirouss Zia, Regular reduction of relativistic theories of gravitation with a quadratic Lagrangian, Phys. Rev. D 32 (1985) 3128.

L. Parker and J.Z. Simon, Einstein equation with quantum corrections reduced to second order, Phys. Rev. D 47 (1993) 1339 and arXiv:gr-qc/9211002 [gr-qc].

[13] T.D. Lee, A relativistic complex pole model with indefinite metric, in Quanta, university of Chicago Press (1970), p. 260.1

[14] J.A. Wheeler and R.P. Feynman, Interaction with the absorber as the mechanism of radiation, Rev. Mod. Phys. 17 (1945) 175;

J.A. Wheeler and R.P. Feynman, Classical electrodynamics in terms of direct interparticle action, Rev. Mod. Phys. 21 (1949) 425.

[15] Check G. 't Hooft and M. Veltman, Diagrammar, CERN report CERN-73-09, §6.1.

[16] N.N. Bogoliubov and D.V. Shirkov, Introduction to the theory of quantized fields, Interscience Publishers, New York, 1959.

[17] H. Lehmann, K. Symanzik and W. Zimmermann, On the formulation of quantized field theories - II, Nuovo Cimento 6 (1957) 319.

[18] D. Anselmi, Fakeons and the classicization of quantum gravity: the FLRW metric, J. High Energy Phys. 04 (2019) 61, 19A1 Renormalization.com and arXiv:1901.09273 [gr-qc]. 
[19] D. Anselmi, Fakeons, microcausality and the classical limit of quantum gravity, Class. and Quantum Grav. 36 (2019) 065010, 18A4 Renormalization.com and arXiv:1809.05037 [hep-th].

[20] C.D. Hoyle, D.J. Kapner, B.R. Heckel, E.G. Adelberger, J.H. Gundlach, U. Schmidt and H.E. Swanson, Sub-millimeter tests of the gravitational inverse-square law, Phys. Rev. D70 (2004) 042004 and arXiv:hep-ph/0405262.

[21] T. Gaumnitz, A. Jain, Y. Pertot, M. Huppert, I. Jordan, F. Ardana-Lamas and H. J. Woerner, Streaking of 43 -attosecond soft-X-ray pulses generated by a passively CEP-stable mid-infrared driver, Opt. Exp. 25 (2017) 27506;

J. Li, X. Ren, Y. Yin, K. Zhao, A. Chew, Y. Cheng, E. Cunningham, Y. Wang, S. Hu, Y. Wu, M. Chini and Z. Chang, 53-attosecond X-ray pulses reach the carbonK-edge, Nature communications 8 (2017) 186.

[22] LIGO, Laser Interferometric Gravitational-Wave Observatory, as reported on the web page https://www.ligo.caltech.edu/page/facts

[23] D. Anselmi, On the nature of the Higgs boson, Mod. Phys. Lett. A 34 (2019) 1950123, 18A7 Renormalization.com and arXiv:1811.02600 [hep-ph].

[24] D. Radice, V. Morozova, A. Burrows, D. Vartanyan and H. Nagakura, Characterizing the gravitational wave signal from core-collapse supernovae, The Astrophysical Journal Letters 876 (2019) L9 and arXiv:1812.07703 [astroph.HE].

[25] M. Fierz and W. Pauli, Proc. Roy. Soc. Lond. A 173, 211 (1939).

[26] See D.G. Boulware and S. Deser, Can gravitation have a finite range?, Phys. Rev. D 6 (1972) 3368, and more recently (among others), N. Arkani-Hamed, H. Georgi and M.D. Schwartz, Effective field theory for massive gravitons and gravity in theory space, Annals Phys. 305 (2003) 96 and arXiv:hep-th/0210184;

P. Creminelli, A. Nicolis, M. Papucci and E. Trincherini, Ghosts in massive gravity, J. High Energy Phys. 0509 (2005) 003 and arXiv:hep-th/0505147. 
[27] C. de Rham and G. Gabadadze, Generalization of the Fierz-Pauli action, Phys. Rev. D 82 (2010) 044020 and arXiv:1007.0443 [hep-th];

C. de Rham, G. Gabadadze and A. Tolley, Resummation of massive gravity, Phys. Rev. Lett. 106 (2011) 231101 and arXiv:1011.1232 [hep-th];

S.F. Hassan and R.A. Rosen, Resolving the ghost problem in non-linear massive gravity, Phys. Rev. Lett. 108 (2012) 041101 and arXiv:1106.3344 [hep-th].

[28] J. Bonifacio and K. Hinterbichler, Unitarization from geometry, J. High Energ. Phys. 12 (2019) 165 and arXiv:1910.04767 [hep-th]. 